\title{
SINGULAR PARABOLIC PARTIAL DIFFERENTIAL EQUATIONS WITH TIME DEPENDENT COEFFICIENTS
}

\author{
BY \\ DORIS S. STOCKTON
}

Introduction. In this paper we consider the general operator

$$
\begin{array}{ll}
\Omega(t)=D_{\mu(t)} D_{\sigma(t)}+c(x, t) & a \leqq t \leqq b, \\
& r_{1} \leqq x \leqq r_{2} .
\end{array}
$$

In (0.1) for each $t, \mu(t)=\mu_{t}$ is a Borel measure defined on the Borel sets of $\left(r_{1}, r_{2}\right), \sigma(t)=\sigma_{t}$ is a strictly increasing function continuous on $\left(r_{1}, r_{2}\right), c(x, t) \leqq 0$, and $c(x, t)$ is continuous in $x$ on $\left[r_{1}, r_{2}\right]$ for each $t \in[a, b]$. The generalized derivatives $D_{\sigma(t)}$ and $D_{\mu(t)} D_{\sigma(t)}$ are defined in $\S 2$. The operator $\Omega(t)$ is, for each $t \in[a, b]$, a linear, but not necessarily bounded, operator. The purpose of this paper is to present conditions on $\mu_{t}$ and $\sigma_{t}$ such that given an initial value for $t=a$ and possible boundary conditions at $r_{1}$ and $r_{2}$ there exists a unique solution of the equation

$$
\begin{array}{ll}
u_{t}(x, t)=D_{\mu(t)} D_{\sigma(t)} u(x, t)+c(x, t) u(x, t) & a \leqq t \leqq b \\
& r_{1} \leqq x \leqq r_{2}
\end{array}
$$

Four types of boundaries, regular, entrance, exit, and natural are considered. These types are defined in $\$ 2$. The boundary classifications of $(0.1)$ for the $\sigma_{t}$ and $\mu_{t}$ independent of $t$ were formulated by $\mathrm{K}$. Itô and used by H. McKean [11] in accordance with W. Feller [4], and the boundary conditions (2.8) are the generalized classical ones.

W. Feller $[3 ; 2]$ has shown that for each $t, \Omega(t)$ is a generalization of the classical differential operator

$$
\Omega(t)=a(x, t) \frac{d^{2}}{d x^{2}}+b(x, t) \frac{d}{d x}+c(x, t) \quad \begin{array}{ll}
a \leqq t \leqq b, \\
r_{1} \leqq x \leqq r_{2}
\end{array}
$$

(See $\S 2$ for the pertinent details of this demonstration.) In $(0.3)$ for each $t, a(x, t)>0$, $a(x, t)$ and $b(x, t)$ are continuous in $x$ on $\left(r_{1}, r_{2}\right), c(x, t) \leqq 0$, and $c(x, t)$ is continuous in $x$ on $\left[r_{1}, r_{2}\right]$. The operators we consider are allowed to be singular in that $a(x, t)$ and $b(x, t)$ are allowed to have discontinuities in $x$ at the boundaries $r_{1}$ and $r_{2}$ and $a(x, t)$ may vanish at the boundaries. The boundary classifications are seen to depend on the behavior of $b^{*}(x, t)=b(x, t) / a(x, t)$ and the boundary con-

Received by the editors August 4, 1961. 
ditions are the classical ones. Our work exhibits conditions on the coefficients of the equation

$$
u_{t}(x, t)=\Omega(t) u(x, t)
$$

which will guarantee the existence of a solution satisfying the classical boundary conditions and uniquely determined by an initial value $u(x, a)$.

Some advantages of the generalized differential operator $(0.1)$ are discussed by $\mathrm{W}$. Feller [2] for the time independent case. The generalization has applications in probability theory and may be used to advantage even for completely classical problems. As W. Feller has pointed out, the generalization achieves a considerable simplification and unification of the theory. In applications one frequently finds that for each $t$ the coefficients of equation (0.4) have jump discontinuities. In such cases one has to piece together solutions in various intervals. But W. Feller [2] has noted that the simple expedient of writing the operator $\Omega(t)$ in the form $(0.1)$ reduces the whole to one single equation and eliminates the laborious piecemeal work. A specific example of such a problem appears as Example 4 in $\$ 8$.

Equation (0.2) can be considered as a special case of the so-called equation of evolution

$$
\frac{d u(t)}{d t}=A(t) u(t) \quad a \leqq t \leqq b
$$

where for each $t \in[a, b], u(t)$ is an element of a Banach space $X$ and $A(t)$ is a linear operator. We shall take as underlying Banach space $X$ either $C\left[r_{1}, r_{2}\right]$ or a subspace of $C\left[r_{1}, r_{2}\right]$ depending on the boundary conditions (Definition (2.1)). The domain of our operator $\Omega(t)$ also depends on the boundary conditions. It is defined as the set of all $f \in X$ which satisfy appropriate boundary conditions and which are such that $\Omega(t) f$ is itself in $X$.

T. Kato [9] has constructed the unique solution to the equation of evolution subject to rather general hypotheses on $A(t)$. In this paper we determine conditions on the scale $\sigma_{t}$ and measure $\mu_{t}$ which guarantee that $\Omega(t)$ satisfies Kato's hypotheses. We supplant Kato's set of hypotheses by a set of conditions which are slightly stronger than Kato's although somewhat more convenient for our purposes. T. Kato [10] has anounced the solution of $(0.5)$ under less stringent conditions than those in [9].

Considerable work has been done by W. Feller [4], J. Elliott [1], and E. Hille [7] in constructing elementary solutions for the classical operator (0.3) where $\Omega(t)=\Omega$ is independent of $t$ and by H. McKean [11] for the generalized operator $(0.1)$ in the $t$ independent case. The elementary solution for $\Omega(t)$ with time dependent coefficients subject to a variety of conditions has been constructed by W. Feller [5]. It appears that our conditions on $b(x, t)$ are less stringent than Feller's as far as $x$ is concerned but are somewhat more restrictive in $t$. 
The method of proof which we use excludes the possibility of using boundary conditions which are dependent on $t$ because in Kato's hypotheses $\operatorname{Dmn} A(t)$ is independent of $t$ and our $\operatorname{Dmn} \Omega(t)$ depends on the boundary conditions. Examples of particular operators $\Omega(t)$ with coefficients $b(x, t)$ having discontinuities at boundaries but which still satisfy the conditions of our main theorem are given in $\S 8$.

In $\$ 1$ we state Kato's theorem and give our conditions which supplant Kato's hypotheses. $\$ 2$ is devoted to preliminary groundwork and the statement of important definitions and conditions. The development of our main theorem for the reduced operator appears in $\S \S 3-5$, and in $\S 6$ we indicate how these proofs go through for the more general case in which $c(x, t)$ is negative. In $\$ 7$ we show in detail how our main theorem applies to the special case of the classical operator, and $\S 8$ consists of several specific examples.

I wish to express my gratitude to Joanne Elliott for suggesting this topic and for her most helpful guidance.

1. Kato's theorem. We wish to employ some of the main results in a paper of T. Kato's [9]. Before summarizing these results, it is convenient to make some preliminary definitions.

Definition 1.1. An operator A in a Banach space $X$ is said to have property $S$ on $X$ : (i) if $A$ is alclosed linear operator with domain dense in $X$; (ii) if the resolvent set of $A$ includes all positive reals; and (iii) if $\left\|(I-\lambda A)^{-1}\right\| \leqq 1$ for $\lambda>0$. Let the following conditions be given:

$\mathrm{K}_{1}$. $A(t)$ is defined for $t \in[a, b]$ and has property $S$ for each $t$.

$\mathrm{K}_{2}$. The domain of $A(t)$ is independent of $t$.

$\mathrm{K}_{3}$. $C(t, s)=[I-A(t)][I-A(s)]^{-1}$ is uniformly bounded; that is, there is an $M>0$ such that $\|C(t, s)\| \leqq M$ for every $s, t$.

$\mathrm{K}_{4} . \quad C(t, s)$ is of bounded variation in $t$ in the sense that there is an $N \geqq 0$ such that

$$
\sum_{j=1}^{n}\left\|C\left(t_{j}, s\right)-C\left(t_{j-1}, s\right)\right\| \leqq N
$$

for every partition $a=t_{0}<t_{1}<\cdots<t_{n}=b$ of the interval $[a, b]$, for some $s$.

$\mathrm{K}_{5}$. $\quad C(t, s)$ is weakly continuous in $t$ for some $s$.

$\mathrm{K}_{6}$. For some $s, C(t, s)$ is weakly differentiable in $t$ and the weak derivative $\partial C(t, s) / \partial t$ is strongly continuous in $t$.

The following theorem represents part of Kato's results:

THEOREM 1.1. If conditions $\mathrm{K}_{1}, \mathrm{~K}_{2}, \cdots, \mathrm{K}_{6}$ are satisfied, then there exists a solution of the equation

$$
d x(t) / d t=A(t) x(t) \quad a \leqq t \leqq b,
$$


where for each $t \in[a, b], x(t)$ is an element of a complex Banach space $X$ and $A(t)$ is a linear operator in $X$. The solution is uniquely determined by the initial value $x(a)$ and is strongly continuously differentiable. Furthermore, if the solution is formally given as $x(t)=U(t, a) x(a)$, then $U(t, t)=I$ and $U(t, r)$ $=U(t, s) U(s, r), \quad r \leqq s \leqq t$.

For our purposes it is convenient to supplant the hypotheses of Kato's theorem with the following set of somewhat stronger conditions:

$\mathrm{C}_{1}$. $A(t)$ is defined for $t \in[a, b]$ and has property $S$ for each $t$.

$\mathrm{C}_{2}$. The domain of $A(t)$ is independent of $t$.

$\mathrm{C}_{3} . \quad C(t, s)=[I-A(t)][I-A(s)]^{-1}$ is strongly continuously differentiable in $t$ for some $s$. That is, for some $s$ the strong derivative $C_{t}(t, s)$ of $C(t, s)$ exists and is strongly continuous in $t$.

This is accomplished by proving the following theorem:

TheOREM 1.2. Conditions $\mathrm{C}_{1}, \mathrm{C}_{2}$ and $\mathrm{C}_{3}$ imply Kato's conditions $\mathrm{K}_{1}, \mathrm{~K}_{2}, \cdots, \mathrm{K}_{6}$.

Proof. It suffices to prove that conditions $C_{1}, C_{2}$ and $C_{3}$ imply Kato's conditions $\mathrm{K}_{3}$ and $\mathrm{K}_{4}$. The uniform boundedness of $C(t, s)$ follows readily (cf. T. Kato [9, pp. 215-216]). Futhermore, $C(t, s)$ is of bounded variation in $t$ since for some fixed $s=s_{0},\left\|C_{t}\left(t, s_{0}\right)\right\|$ is uniformly bounded in $t$.

2. Preliminary definitions. Let $x$ be an element of the open interval $\left(r_{1}, r_{2}\right)$ and for convenience let $0 \in\left(r_{1}, r_{2}\right)$. Let $t \in[a, b]$ for $a \geqq 0$. For each $t$ let $\sigma_{t}(x)$ $=\sigma(x, t)$ be a strictly increasing continuous function-that is, an arbitrary scale. For each $t$ let $\mu_{t}$ be a Borel measure defined on the class of Borel sets of $\left(r_{1}, r_{2}\right)$ and let $\mu(x, t)=\mu_{t}((0, x])$ for $x>0, \mu(x, t)=\mu_{t}((x, 0])$ for $x<0$ and $\mu(0, t)=\mu_{t}(\{0\})$. $\mu(x, t)$ is right continuous in $x$ for each $t . \mu(x, t)$ is monotone, and we assume $\mu(x, t)$ to be strictly increasing for $x>0$ and strictly decreasing for $x<0$ so that its discontinuities form a Borel set of measure zero.

Let $f \in C\left[r_{1}, r_{2}\right]$. For each $t$ and $x \in\left(r_{1}, r_{2}\right)$ define

$$
D_{\sigma(t)} f(x)=\lim _{h \rightarrow 0} \frac{f(x+h)-f(x)}{\sigma_{t}(x+h)-\sigma_{t}(x)}
$$

and at points of continuity of $\mu(x, t)$ define

and

$$
D_{\mu(t)}^{+} D_{\sigma(t)} f(x)=\lim _{h \rightarrow 0^{+}} \frac{D_{\sigma(t)} f(x+h)-D_{\sigma(t)} f(x)}{\mu_{t}((x, x+h))}
$$

$$
D_{\mu(t)}^{-} D_{\sigma(t)} f(x)=\lim _{h \rightarrow 0^{-}} \frac{D_{\sigma(t)} f(x+h)-D_{\sigma(t)} f(x)}{-\mu_{t}((x+h, x))} .
$$

If $D_{\mu(t)}^{+} D_{\sigma(t)} f(x)=D_{\mu(t)}^{-} D_{\sigma(t)} f(x)$, then define

$$
D_{\mu(t)} D_{\sigma(t)} f(x)=D_{\mu(t)}^{+} D_{\sigma(t)} f(x)=D_{\mu(t)}^{-} D_{\sigma(t)} f(x) .
$$


At points of discontinuity of $\mu(x, t)$ define

$$
D_{\mu(t)} D_{\sigma(t)} f(x)=\frac{D_{\sigma(t)} f(x+)-D_{\sigma(t)} f(x-)}{\mu(x+, t)-\mu(x-, t)} .
$$

Of course $D_{\sigma(t)} f$ is continuous with respect to $\mu_{t}$ when $D_{\mu(t)} D_{\sigma(t)} f$ exists. The only possible points of discontinuity of $D_{\sigma(t)} f$ are the points of discontinuity of $\mu_{t}$. For convenience we will assume that $D_{\sigma(t)} f(x)$ is continuous to the right for $x \in\left[r_{1}, r_{2}\right]$.

Let $\Omega(t) f(x)=D_{\mu(t)} D_{\sigma(t)} f(x)+c(x, t) f(x)$, where for each $t, c(x, t) \leqq 0$ and is continuous in $x$ on the closed interval $\left[r_{1}, r_{2}\right]$. The domain of $\Omega(t)$ will be defined below. We wish to consider the equation

$$
u_{t}(x, t)=\Omega(t) u(x, t) .
$$

To that end let us classify boundaries and define the classical boundary conditions.

As a preliminary to classifying the boundaries, let us imitate H. P. McKean [11] in defining $\left({ }^{1}\right)$

$$
J_{i}(t)=(-1)^{i} \int_{0}^{r i}(1-c(x, t)) \mu(x, t) d \sigma_{t}
$$

and

$$
K_{i}(t)=(-1)^{i} \int_{0}^{r i}(1-c(x, t))|\sigma(x, t)| d \mu_{t} \quad \text { for } i=1,2 .
$$

For future use note that for each $t$

and

$$
\begin{aligned}
K_{i}(t) & <+\infty \rightarrow K_{i}^{0}(t)=(-1)^{i} \int_{0}^{r i}|\sigma(x, t)| d \mu_{t} \\
& <+\infty \rightarrow(-1)^{i} \int_{0}^{r i} d \mu_{t}<+\infty
\end{aligned}
$$

$$
\begin{gathered}
J_{i}(t)<+\infty \rightarrow J_{i}^{0}(t)=(-1)^{i} \int_{0}^{r i} \mu(x, t) d \sigma_{t} \\
<+\infty \rightarrow(-1)^{i} \int_{0}^{r t} d \sigma_{t}<+\infty .
\end{gathered}
$$

Here the $K_{i}(t), K_{i}^{0}(t), J_{i}(t)$ and $J_{i}^{0}(t)$ are all non-negative for $i=1,2$; and of course if $c(x, t) \equiv 0, K_{i}(t)=K_{i}^{0}(t)$ and $J_{i}(t)=J_{i}^{0}(t)$. As suggested by W. Feller [4] and H. P. McKean and K. Itô [11], we now classify boundaries as follows: where $i=1$ or 2 ,

(1) Throughout this paper the notation $\int^{x} f(x, t) d \mu_{t}$ is used for the integral over the semi-open interval $\left(x_{1}, x_{2}\right]$. 
$\left[r_{i}\right.$ is regular] $\leftrightarrow$ [for each $t, J_{i}(t)<+\infty$ and $\left.K_{i}(t)<+\infty\right]$, $\left[r_{i}\right.$ is exit] $\leftrightarrow$ [for each $t, J_{i}(t)<+\infty$ and $K_{i}(t)=+\infty$ ], $\left[r_{i}\right.$ is entrance $] \leftrightarrow$ [for each $t, J_{i}(t)=+\infty$ and $\left.K_{i}(t)<+\infty\right]$, [ri $r_{i}$ is natural] $\leftrightarrow$ [for each $t, J_{i}(t)=+\infty$ and $\left.K_{i}(t)=+\infty\right]$.

Define the classical boundary conditions as follows: where $i=1$ or 2 and $p_{i} \in[0,1]$,

$$
\begin{aligned}
\lim _{x \rightarrow r_{i}}\left\{\left(1-p_{i}\right) u(x, t)+(-1)^{i} p_{i} D_{\sigma(t)} u(x, t)\right\} & =0 \text { for } r_{i} \text { regular, } \\
\lim _{x \rightarrow r i} u(x, t) & =0 \text { for } r_{i} \text { exit, } \\
\lim _{x \rightarrow r_{i}} D_{\sigma(t)} u(x, t) & =0 \text { for } r_{i} \text { entrance, } \\
\lim _{x \rightarrow r i} u(x, t) & =0 \text { for } r_{i} \text { natural. }
\end{aligned}
$$

Definition 2.1. For each $t$ we associate with the operator $\Omega(t)$ a Banach space $X$ given by: (i) $C\left[r_{1}, r_{2}\right]$ if $r_{i}(i=1,2)$ is entrance or regular with $p_{i} \neq 0$ in (2.10); (ii) the subspace of $C\left[r_{1}, r_{2}\right]$ of functions vanishing at $r_{i}$ if $r_{i}$ is regular with $p_{i}=0$ in (2.10) or if $r_{i}$ is exit or natural.

We may now precisely define the operator $\Omega(t)$ as follows: if

$$
\Omega(t)=D_{\mu(t)} D_{\sigma(t)}+c(x, t),
$$

then Domain of $\Omega(t)$ is the set of all $u \in X$ such that $u(x)$ satisfies the boundary conditions prescribed for the type of boundaries in question and such that $\left({ }^{2}\right)$ $\Omega(t) u \in X$.

Special Case. For each $t$ the operator

$$
A(t)=a(x, t) \frac{d^{2}}{d x^{2}}+b(x, t) \frac{d}{d x}+c(x, t),
$$

is a special case of (2.11) when $a(x, t)>0, a(x, t)$ and $b(x, t)$ are continuous for $x \in\left(r_{1}, r_{2}\right)$, and $c(x, t) \leqq 0$ and continuous on $\left[r_{1}, r_{2}\right]$. This was shown by $\mathrm{W}$. Feller in his paper On second order differential operators [3]. (See also W. Feller [2].) In particular consider the so-called reduced operator

$$
A(t)=a(x, t) \frac{d^{2}}{d x^{2}}+b(x, t) \frac{d}{d x} .
$$

If $b(x, t) / a(x, t)=b^{*}(x, t)$, and if

$$
B(x, t)=\int_{0}^{x} b^{*}(s, t) d s,
$$

then by choosing

$$
\sigma(x, t)=\int_{0}^{x} e^{-B(s, t)} d s
$$

(2) $\Omega(t) u \in X$ by virtue of the fact that $\Omega(t) u \in C\left(r_{1}, r_{2}\right), \lim _{x \rightarrow r} \Omega(t) u(x)=L_{t}$ exists finite, and $\Omega(t) u\left(r_{i}\right)$ is defined as $L_{i}$. 
and

$$
\mu(x, t)= \pm \int_{0}^{x} \frac{1}{a(s, t)} e^{B(s, t)} d s,
$$

according as $x>0$ or $x<0$, the reduced operator is readily seen to be of the form $D_{\mu(t)} D_{\sigma(t)}$. Examples of the reduced operator with specific coefficients will be presented in $\S 8$.

The following lemma is of fundamental importance. It was proved for the operator

$$
A(t)=a(x, t) \frac{d^{2}}{d x^{2}}+b(x, t) \frac{d}{d x}+c(x, t)
$$

by W. Feller [4]; H. P. McKean [11] used direct analogy with Feller's proof to establish the lemma for the operator (2.11).

Lemma 2.1. For each $t \in[a, b]$ let $\sigma_{t}(x)=\sigma(x, t)$ be a strictly increasing continuous function. For each $t \in[a, b]$ let $\mu_{t}$ be a Borel measure defined on the Borel sets of $\left(r_{1}, r_{2}\right)$, and let $\mu(x, t)=\mu_{t}((0, x])$ for $x>0$ and $\mu(x, t)=\mu_{t}((x, 0])$ for $x<0$. Assume $\mu(x, t)$ is strictly increasing for $x>0$ and strictly decreasing for $x<0$. Let $c(x, t) \leqq 0$ be continuous in $x$ on $\left[r_{1}, r_{2}\right]$ for each $t \in[a, b]$. Then in all boundary classifications condition $\mathrm{C}_{1}$ holds for the operator (2.11), that is, (2.11) is defined for $t \in[a, b]$ and has property $S$ on $X$ for each $t$.

Throughout this entire paper we shall assume that the following condition is true:

A. For each $t \in[a, b], \mu_{t}$ and $\sigma_{t}$ are differentiable with respect to $\mu_{s}$ and $\sigma_{s}$ respectively. That is, the derivatives

$$
\begin{aligned}
& \frac{d \sigma_{t}}{d \sigma_{s}}=\lim _{h \rightarrow 0} \frac{\sigma_{t}(x+h)-\sigma_{t}(x)}{\sigma_{s}(x+h)-\sigma_{s}(x)}, \\
& \frac{d \mu_{t}}{d \mu_{s}}=\lim _{h \rightarrow 0} \frac{\mu_{t}((x, x+h))}{\mu_{s}((x, x+h))} \text { at points of continuity of } \mu(x, s),
\end{aligned}
$$

and

$$
\frac{d \mu_{t}}{d \mu_{s}}=\frac{\mu\left(x^{+}, t\right)-\mu\left(x^{-} t\right)}{\mu\left(x^{+}, s\right)-\mu\left(x^{-}, s\right)} \text { at points of discontinuity of } \mu(x, s) \text { exist. }
$$

In addition to condition $\mathrm{A}$ stated above, future reference will be made to several other conditions on $\mu_{t}$ and $\sigma_{t}$. These conditions are stated in terms of $\left({ }^{3}\right)$

$$
\phi(x, s, t)=\frac{d \mu_{t}}{d \mu_{s}} \cdot D \mu_{(t)}\left(\frac{d \sigma_{t}}{d \sigma_{s}}\right)=D \mu_{(s)} D \sigma_{(s)} \sigma(t, x),
$$

(3) If for $t, s \in[a, b], f(x, t, s)$ is continous in $x$ on $\left[r_{1}, r_{2}\right]$, then for each $t, s, f(x, t, s)$ defines a vectorvalued function from $a \leqq s \leqq b, a \leqq t \leqq b$ to $C\left[r_{1}, r_{2}\right]$; and we denote the value of the function by $f(\cdot, t, s)$ so $f(\cdot, t, s)$ is an element of $C\left[r_{1}, r_{2}\right]$. In particular, (2.16) and (2.17) define the real-valued functions $\phi(\cdot, s, t)$ and $\psi(\cdot, s, t)$. 


$$
\begin{aligned}
\psi(x, s, t) & =\frac{d \mu_{t}}{d \mu_{s}} \cdot \frac{d \sigma_{t}}{d \sigma_{s}} \\
C(t, s) & =[I-\Omega(t)][I-\Omega(s)]^{-1}, \\
F(s, x) & =[I-\Omega(s)]^{-1} f(x) .
\end{aligned}
$$

For the convenience of the reader we shall conclude this section with a list of the other conditions on $\mu_{t}$ and $\sigma_{t}$ to which we shall refer in the sequel.

B. For each $s \in[a, b], \mu_{s}$ is differentiable with respect to $\sigma_{s}$, that is,

$$
\frac{d \mu_{s}}{d \sigma_{s}}=\lim _{h \rightarrow 0} \frac{\mu_{s}(x, x+h)}{\sigma_{s}(x+h)-\sigma_{s}(x)}
$$

exists. (Note that $B$ implies that $\mu_{t}$ is a continuous measure.)

C. $\psi(\cdot, s, t) \in C\left[r_{1}, r_{2}\right]$ for each $t, s \in[a, b] . \phi(\cdot, s, t) \in X$ for each $t, s \in[a, b] .\left(^{4}\right)$

D. $c(\cdot, t)$ is nonpositive for each $t$ and $c(\cdot, t)$ is a strongly continuously differentiable function from $[a, b]$ to $X .\left({ }^{5}\right)$

E. For some $s=s_{0}, \psi\left(\cdot, t, s_{0}\right)$ and $\phi\left(\cdot, t, s_{0}\right)$ are strongly continuously differentiable functions from $[a, b]$ to $X$.

$\mathrm{F}_{i} . \quad(i=1,2) . \lim _{x \rightarrow r i} \phi(x, s, t) \mu(x, t)=0$ for each $s, t \in[a, b]$.

$\mathrm{F}_{3}$. For each $s, t, d \sigma_{t} / d \sigma_{s}$ is continuous at points of discontinuity of $\mu(\cdot, t)$. (Note that since condition $\mathrm{A}$ is assumed, if $\phi(\cdot, s, t) \in X$, condition $\mathrm{F}_{3}$ implies that $d \sigma_{t} / d \sigma_{s}$ is continuous everywhere on $\left[r_{1}, r_{2}\right]$.)

$\mathrm{G}_{i}$. There exist positive constants $m$ and $M$ independent of $s$ such that for some $x_{i} \in\left[0, r_{i}\right), m<\left|\sigma_{s}\left(r_{i}\right)-\sigma_{s}\left(x_{i}\right)\right|<M$.

H. $\mathrm{E}$ is true and $\phi^{*}(\cdot, t, s)=\phi(\cdot, t, s) \mu(\cdot, s)$ is a strongly continuously differentiable function from $[a, b]$ to $X$ for some $s=s_{0}$ with $\phi_{t}^{*}\left(\cdot, t, s_{0}\right)=$ $\phi_{t}\left(\cdot, t, s_{0}\right) \mu\left(\cdot, s_{0}\right)$.

J. $\quad E$ is true and $\phi_{t}\left(\cdot, t, s_{0}\right) \mu\left(\cdot, s_{0}\right) \in X$ for each $t .\left(^{6}\right)$

3. Conditions under which domain of $D_{\mu(t)} D_{\sigma(t)}$ is independent of $t$. In this section and through $\S 5$ we shall assume that $c(x, t) \equiv 0$ so that $(0.1)$ is the reduced operator $\Omega(t)=D_{\mu(t)} D_{\sigma(t)}$. Let us now exhibit conditions under which the domain of $\Omega(t)=D_{\mu(t)} D_{\sigma(t)}$ is independent of $t$ for various types of boundary combinations.

(4) A function $g$ will be considered to be in $X$ if $g \in C\left[r_{1}, r_{2}\right]$ and if $\lim _{x \rightarrow r} g(x)$ exists and has the appropriate value as specified in Definition 2.1.

(5) The vector-valued function $c(\cdot, t)$ is a strongly differentiable function from $[a, b]$ to $X$ if, and only if, there exists a function $c_{t}(\cdot, t) \in X$ such that $\lim _{h \rightarrow 0} \max _{x \in\left[r_{1}, r_{2}\right]} \mid(c(x, t+h)$ $-c(x, t)) / h-c_{t}(x, t) \mid=\lim _{h \rightarrow 0}\left\|(c(\cdot, t+h)-c(\cdot, t)) / h-c_{t}(\cdot, t)\right\|=0 . c_{t}(\cdot, t)$ is strongly continuous if, and only if $\lim _{t \rightarrow t_{0}} \max _{x \in\left[r_{1}, r_{2}\right]}\left|c_{t}(x t)-c_{t}\left(x, t_{0}\right)\right|=\lim _{t \rightarrow t_{0}}\left\|c_{t}(\cdot, t)-c_{t}\left(\cdot, t_{0}\right)\right\|$ $=0$.

(6) Note that $\mathrm{H} \rightarrow \mathrm{J}$ and $\mathrm{H} \rightarrow \mathrm{E}$. 
LEMMA 3.1. If both boundaries are regular, both are entrance, or one boundary is regular and one is entrance, conditions $\mathrm{B}, \mathrm{C}$ and $\mathrm{F}_{3}$ imply condition $\mathrm{C}_{2}$ : the domain of $\Omega(t)=D_{\mu(t)} D_{\sigma(t)}$ is independent of $t$.

Proof. It suffices to show that $u \in \operatorname{Dmn} \Omega(t)$ implies that $u \in \operatorname{Dmn} \Omega(s)$ for $t \neq s$ and $t, s \in[a, b]$. Let

$$
\Omega(t) u=f \in X
$$

We have

$$
\begin{aligned}
\Omega(s) u & =D_{\mu(s)} D_{\sigma(s)} u \\
& =\frac{d \mu_{t}}{d \mu_{s}} \cdot D_{\mu(t)}\left(D_{\sigma(s)} u\right) \\
& =\frac{d \mu_{t}}{d \mu_{s}} \cdot D_{\mu(t)}\left(D_{\sigma(t)} u \cdot \frac{d \sigma_{t}}{d \sigma_{s}}\right) .
\end{aligned}
$$

So

$$
\Omega(s) u(x)=\psi(x, s, t) \cdot \Omega(t) u(x)+\phi(x, s, t) \cdot D_{\sigma(t)} u(x)
$$

or

$$
\Omega(s) u=\psi(\cdot, s, t) f+\phi(\cdot, s, t) D_{\sigma(t)} u .
$$

Since $\psi(\cdot, s, t) \in C\left[r_{1}, r_{2}\right]$ and $f \in X$ by hypothesis, the problem of showing that the $\operatorname{Dmn} \Omega(t)$ is independent of $t$ reduces to that of showing that $\phi(\cdot, s, t) D_{\sigma(t)} u$ is an element of $X$. Let us obtain a convenient expression for $\phi(x, s, t) D_{\sigma(t)} u(x)$ by considering the differential equation (3.1). This equation may be written

$$
D_{\mu(t)} D_{\sigma(t)} u(x)=f(x) \text {. }
$$

We may integerate over $(0, x]$ where $x \in\left(r_{1}, r_{2}\right)$ and obtain

$$
\phi(x, s, t) D_{\sigma(t)} u(x)=\phi(x, s, t) D_{\sigma(t)} u(0)+\phi(x, s, t) \int_{0}^{x} f(y) d \mu_{t} .
$$

Since $f \in X$, the function defined for each $s, t$ by the second term on the right of (3.6) will be continuous on the open interval $\left(r_{1}, r_{2}\right)$ if we can show that

$$
\phi(\cdot, s, t) \mu(\cdot, t) \in C\left(r_{1}, r_{2}\right)
$$

for each $s, t$. With that aim in mind, note that $\phi(\cdot, s, t) \mu(\cdot, t)$ is continuous on $\left(r_{1}, r_{2}\right)$ except possibly at points of discontinuity of $\mu(\cdot, t)$. These discontinuities form a countable set of jumps and since $d \mu_{t} / d \mu_{s}$ exists, for each $t \neq s \mu(\cdot, t)$ and $\mu(\cdot, s)$ are discontinuous at the same points. By definition

$$
\begin{aligned}
\phi(x, s, t) & =\frac{d \mu_{t}}{d \sigma_{s}} \cdot D_{\mu(t)}\left(\frac{d \sigma_{t}}{d \sigma_{s}}\right) \\
& =D_{\mu(s)}\left(\frac{d \sigma_{t}}{d \sigma_{s}}\right)
\end{aligned}
$$


but by hypothesis $d \sigma_{t} / d \sigma_{s}$ is continuous at points of discontinuity of $\mu(\cdot, t)$ and hence of $\mu(\cdot, s)$ so $\phi(x, s, t)=0$ at such points. It follows that for each $s, t$, $\phi(\cdot, s, t) \mu(\cdot, t) \in C\left(r_{1}, r_{2}\right)$ and the function defined by the second term on the right of (3.6) is in $C\left(r_{1}, r_{2}\right)$. The function defined for each $s, t$ by the first term on the right of (3.6) is in $X$ by hypothesis. Consequently $\phi(\cdot, s, t) D_{\sigma(t)} u$ is continuous on the open interval. To show that $\phi(\cdot, s, t) D_{\sigma(t)} u$ is actually in $X$, we have only to show that $\lim _{x \rightarrow r_{i}} \int_{0}^{x} f(y) d \mu_{t}$ exists. This is clearly true since $K_{i}(t)<+\infty$ for $r_{i}$ regular or entrance; and $K_{i}(t)<+\infty$ implies $(-1)^{i} \int_{0}^{r_{i}} d \mu_{t}<+\infty$ which in turn implies that for $f \in C\left[r_{1}, r_{2}\right],(-1)^{i} \int_{0}^{r t}|f(y)| d \mu_{t}<+\infty$. Thus $\phi(\cdot, s, t) D_{\sigma(t)} u \in X$, and it follows that $\Omega(s) u \in X$.

The argument just used to show that $\phi(\cdot, s, t) D_{\sigma(t)} u$ is in $X$ breaks down if $r_{i}$ is an exit or natural boundary since $K_{i}(t)=+\infty$ in these cases. Consequently we shall need to impose an additional condition on $\phi(\cdot, s, t)$ for exit or natural boundaries. This gives rise to the following lemma.

LEMMA 3.2. (a) If both boundaries are exit, both are natural, or one boundary $s$ natural and one is exit, conditions $\mathrm{B}, \mathrm{C}, \mathrm{F}_{1}, \mathrm{~F}_{2}$ and $\mathrm{F}_{3}$ imply condition $\mathrm{C}_{2}$ : the domain of $\Omega(t)=D_{\mu(t)} D_{\sigma(t)}$ is independent of $t$.

(b) If $r_{i}$ is natural or exit and if $r_{j}(i \neq j ; i, j=1,2)$ is regular or entrance, conditions $\mathrm{B}, \mathrm{C}, \mathrm{F}_{i}$, and $\mathrm{F}_{3}$ imply condition $\mathrm{C}_{2}$ : the domain of $\Omega(t)=D_{\mu(t)} D_{\sigma(t)}$ is independent of $t$.

Proof. For $u \in \operatorname{Dmn} \Omega(t)$, let $\Omega(t) u=f \in X$. Then as in the proof of Lemma 3.1, we have equations (3.4) and (3.6). Again examination of the right member of (3.6) shows that we have $\phi(\cdot, s, t) D_{\sigma(t)} u$ continuous on the open interval. The function defined for each $s, t$ by the first term on the right of (3.6) is an element of $X$ by hypothesis. Moreover, if $\mathrm{F}_{i}$ is true, for each $s, t$ the function defined by the second term on the right of (3.6) is also an element of $X$. Thus in all boundary classifications, conditions $\mathrm{B}, \mathrm{C}, \mathrm{F}_{1}, \mathrm{~F}_{2}$ and $\mathrm{F}_{3}$ imply that the domain of $\Omega(t)$ $=D_{\mu(t)} D_{\sigma(t)}$ is independent of $t$.

If $r_{j}$ happens to be regular or entrance and $r_{i}(i \neq j)$ is exit or natural, however, we need only the conditions $B, C, F_{i}$ and $F_{3}$ to prove the domain is independent of $t$ since we saw in Lemma 3.1 that $\phi(\cdot, s, t) D_{\sigma(t)} u$ is forced to be continuous at a regular or entrance endpoint by conditions $B$ and $C$.

4. Conditions implying $C_{3}$ for regular and/or entrance boundaries with reduced operator $\Omega(t)=D_{\mu(t)} D_{\sigma(t)}$. Now let us consider the operator $C(t, s)=[I-\Omega(t)]$ - $[I-\Omega(s)]^{-1}$ and for various combinations of boundaries let us exhibit conditions implying condition $\mathrm{C}_{3}: C(t, s)$ is strongly continuously differentiable in $t$ for some $s$ when $\Omega(t)$ is the reduced operator $D_{\mu(t)} D_{\sigma(t)}$.

First note that if $\operatorname{Dmn} \Omega(t)$ is independent of $t$, then for each $t$ and each $s$, $C(t, s)$ is an element of $E(X)$, the Banach algebra of endomorphisms on $X$. The boundedness follows from Lemma 2 of T. Kato [9], and $f \in X$ implies that 
$C(t, s) f \in X$ since $F(s, \cdot)=[I-\Omega(s)]^{-1} f$ is an element of $\operatorname{Dmn} \Omega(s)$. Also note that for $f \in X, C(t, s) f$ can be expressed as follows:

$$
\begin{aligned}
C(t, s) f & =F(s, \cdot)-\psi(\cdot, t, s) \Omega(s) F(s, \cdot)-\phi(\cdot, t, s) D_{\sigma(s)} F(s, \cdot) \\
& =F(s, \cdot)-\psi(\cdot, t, s)[F(s, \cdot)-f]-\phi(\cdot, t, s) D_{\sigma(s)} F(s, \cdot) .
\end{aligned}
$$

Assuming that the strong derivatives $\psi_{t}(\cdot, t, s)$ and $\phi_{t}(\cdot, t, s)$ of $\psi(\cdot, t, s)$ and $\phi(\cdot, t, s)$ exist for some fixed $s$, define the operator $C_{t}(t, s)$ so that for the fixed $s$,

$$
C_{t}(t, s) f=-\psi_{t}(\cdot, t, s)[F(s, \cdot)-f]-\phi_{t}(\cdot, t, s) D_{\sigma(s)} F(s, \cdot) \text {. }
$$

The object of the next two lemmas is to show conditions under which this operator $C_{t}(t, s)$ is actually the strong derivative of $C(t, s)$ with respect to $t$ for the fixed $s$ and is strongly continuous.

LEMMA 4.1. If $\Omega(t)=D_{\mu(t)} D_{\sigma(t)}$ and both boundaries are regular, both are entrance or one boundary is regular and one is entrance, conditions $\mathrm{A}, \mathrm{B}, \mathrm{C}$, $\mathrm{E}, \mathrm{F}_{3}$ and $\mathrm{J}$ imply condition $\mathrm{C}_{3}: C(t, s)=[I-\Omega(t)][I-\Omega(s)]^{-1}$ is strongly continuously differentiable in $t$ for some s.

Proof. Fix $s$ at the value for which $\psi_{t}(\cdot, t, s)$ and $\phi_{t}(\cdot, t, s)$ exist as assumed in E. First we shall show that $C_{t}(t, s)$ as defined in (4.3) is actually an endomorphism on $X$, for each $t$ and some $s$. Then we shall show that $C_{t}(t, s)$ is the strong derivative of $C(t, s)$ with respect to $t$ for our fixed $s$ and is strongly continuous.

To show that $C_{t}(t, s)$ is bounded, consider equation (4.3). Notice that the first term on the right of (4.3) is bounded for each $t$ and the fixed $s$. Indeed $\left\|\psi_{t}(\cdot, t, s)\right\|$ is bounded in $t$ and for $f \in X$,

$$
\left\|\psi_{t}(\cdot, t, s)[F(s, \cdot)-f]\right\| \leqq 2\left\|\psi_{t}(\cdot, t, s)\right\| \cdot\|f\|
$$

inasmuch as $\Omega(s)$ has property $S$ on $X$. To complete the proof that $C_{t}(t, s)$ is bounded for each $t$, we show that for each $t$ and our fixed $s$ there exists a constant $K(s)>0$ such that

$$
\left\|\phi:(\cdot, t, s) D_{\sigma(s)} F(s, \cdot)\right\| \leqq K(s)\|f\| .
$$

To this end let us obtain a convenient expression for $D_{\sigma(s)} F(s, \cdot)$ by integrating the equation

$$
D_{\mu(s)} D_{\sigma(s)} F(s, x)=F(s, x)-f(x)
$$

over $\left(x, r_{2}\right]$ (analogous considerations may be made for $r_{1}$ ). We have

$$
D_{\sigma(s)} F(s, x)=\lim _{x \rightarrow r_{2}} D_{\sigma(s)} F(s, x)-\int_{x}^{r_{2}}[F(s, y)-f(y)] d \mu_{s} .
$$

In (4.7) $\lim _{x \rightarrow r_{2}} D_{\sigma(s)} F(s, x)$ exists because $r_{2}$ is either regular or entrance. There exists a positive constant $N_{2}(s)$ such that for our fixed $s$, the second term on the right of (4.7) clearly satisfies the inequality

$$
\left|\int_{x}^{r_{2}}[F(s, y)-f(y)] d \mu_{s}\right| \leqq N_{2}(s)\|f\|
$$


inasmuch as $\|F(s, \cdot)\| \leqq\|f\|$ and $(-1)^{t} \int_{0}^{r t} d \mu_{s}$ exists finite. To show that the first term on the right of (4.7) satisfies a similar inequality, let us obtain an expression for $D_{\sigma(s)} F(s, x)$ by writing out the solution of the nonhomogeneous equation (4.6) in terms of the Green function and then differentiating with respect to $\sigma_{s}$. From H. McKean [11] (analogue of W. Feller [4]) we have

$$
F(s, x)=\int_{r_{1}}^{r_{2}} G(x, y ; s) f(y) d \mu_{s}
$$

where

$$
\begin{aligned}
G(x, y ; s) & =\frac{u_{1}(x, s) u_{2}(y, s)}{\omega\left(u_{2}(y, s), u_{1}(y, s)\right)} & & \text { for } y \geqq x \\
& =\frac{u_{1}(y, s) u_{2}(x, s)}{\omega\left(u_{2}(y, s), u_{1}(y, s)\right)} & & \text { for } y<x .
\end{aligned}
$$

Here (i) $u_{1}$ is positive strictly increasing and $u_{2}$ is positive strictly decreasing, (ii) $u_{1}$ and $u_{2}$ are solutions of the homogeneous equation $[I-\Omega(s)] u=0$, (iii) $u_{i}$ satisfies regular or entrance boundary conditions depending on whether $r_{i}$ is regular or entrance, and (iv) $\lim _{x \rightarrow r_{i}} D_{\sigma(s)} u_{i}(x, s)<+\infty$. Moreover from W. Feller [3] we learn that the wronskian $\omega\left(u_{2}(y, s), u_{1}(y, s)\right)=D_{\sigma(s)} u_{1}(x) u_{2}$ $-u_{1} D_{\sigma(s)} u_{2}(x)$ is a positive constant $k(s)$. Without loss of generality we can consider the wronskian $\omega\left(u_{2}, u_{1}\right)$ to be normed so that $k(s)=1$. Therefore,

$$
F(s, x)=u_{2}(x, s) \int_{r_{1}}^{x} u_{1}(y, s) f(y) d \mu_{s}+u_{1}(x, s) \int_{x}^{r_{2}} u_{2}(y, s) f(y) d \mu_{s} .
$$

Now we can compute $D_{\sigma(s)} F(s, x)$ from (4.11) as follows:

$$
D_{\sigma(s)} F(s, x)=D_{\sigma(s)} u_{2}(x, s) \int_{r_{1}}^{x} u_{1}(y, s) f(y) d \mu_{s}+D_{\sigma(s)} u_{1}(x, s) \int_{x}^{r_{2}} u_{2}(y, s) f(y) d \mu_{s} .
$$

An analysis of all the possible choices of the constant $p_{2}$ in the definition of the boundary conditions $\left({ }^{7}\right)$ shows that if $r_{2}$ is regular and $r_{1}$ is entrance or regular, either

$$
\lim _{x \rightarrow r_{2}} D_{\sigma(s)} F(s, x)=0
$$

or

$$
\lim _{x \rightarrow r_{2}} D_{\sigma(s)} F(s, x)=-n \int_{r_{1}}^{r_{2}} u_{1}(y, s) f(y) d \mu_{s}
$$

where $n>0$, and $u_{1}(x, s)$ is a positive strictly increasing solution of $y-\Omega(s) y=0$ such that $\lim _{x \rightarrow r_{2}} u_{1}(x, s)$ and $\lim _{x \rightarrow r_{2}} D_{\sigma(s)} u_{1}(x, s)$ are both finite. In either case if $r_{2}$ is regular, there exists a positive constant $N_{1}(s)$ such that

(7) Complete details are given in [12]. 


$$
\left|\lim _{x \rightarrow r_{2}} D_{\sigma(s)} F(s, x)\right| \leqq N_{1}(s)\|f\|
$$

for our fixed $s$.

It follows from (4.7), (4.8), and (4.15) that if $r_{2}$ is regular and $r_{1}$ is entrance or regular, there exists $N(s)>0$ such that for our fixed $s$,

$$
\left\|D_{\sigma(s)} F(s, \cdot)\right\| \leqq N(s)\|f\| .
$$

Consequently the boundedness condition (4.5) holds and so for $r_{2}$ regular and $r_{1}$ entrance or regular $C_{t}(t, s)$ is bounded for each $t$ and our fixed $s$. To complete the proof of the boundedness of $C_{t}(t, s)$ it remains to consider the case in which $r_{2}$ is an entrance boundary. In this case, since $F(s, \cdot) \in \operatorname{Dmn} \Omega(s)$ and hence satisfies appropriate boundary conditions, $\lim _{x \rightarrow r_{2}} D_{\sigma(s)} F(s, x)=0$ and again the boundedness condition (4.5) holds and forces $C_{t}(t, s)$ to be bounded for each $t$ and our fixed $s$.

To see that $C_{t}(t, s)$ is an endomorphism on $X$, it remains to show that for $f \in X, C_{t}(t, s) f \in X$. We see from (4.3) that, to show this, it suffices to note that $\phi_{r}(\cdot, t, s) D_{\sigma(s)} F(s, \cdot) \in X$.

To demonstrate that $C(t, s)$ is strongly continously differentiable in $t$ for some $s$, we must show that $C_{t}(t, s)$ actually is the strong derivative of $C(t, s)$ with respect to $t$ for our fixed $s$ and we must show that $C_{t}(t, s)$ is strongly continuous in $t$ for our fixed $s$. In other words we must show that

and that

$$
\lim _{h \rightarrow 0}\left\|\left[\frac{C(t+h, s)-C(t, s)}{h}-C_{t}(t, s)\right] f\right\|=0
$$

$$
\lim _{t \rightarrow t_{0}}\left\|\left[C_{t}(t, s)-C_{t}\left(t_{0}, s\right)\right] f\right\|=0
$$

both for all $f \in X$ and the fixed s. Computing from (4.2) and (4.3) we have for any $f \in X$,

$$
\begin{aligned}
& 0 \leqq\left\|\left[\frac{C(t+h, s)-C(t, s)}{h}-C_{t}(t, s)\right] f\right\| \\
\leqq & \left\|\frac{\psi(\cdot, t+h, s)-\psi(\cdot, t, s)}{h}-\psi_{t}(\cdot, t, s)\right\|\|F(s, \cdot)-f\| \\
& +\left\|\frac{\phi(\cdot, t+h, s)-\phi(\cdot, t, s)}{h}-\phi_{t}(\cdot, t, s)\right\|\left\|D_{\sigma(s)} F(s, \cdot)\right\|
\end{aligned}
$$

and

$$
\begin{aligned}
0 \leqq & \left\|\left[C_{t}(t, s)-C_{t}\left(t_{0}, s\right)\right] f\right\| \\
\leqq & \left\|\psi_{t}(\cdot, t, s)-\psi_{t}\left(\cdot, t_{0}, s\right)\right\|\|F(s, \cdot)-f\| \\
& +\left\|\phi_{t}(\cdot, t, s)-\phi_{t}\left(\cdot, t_{0}, s\right)\right\|\left\|D_{\sigma(s)} F(s, \cdot)\right\| .
\end{aligned}
$$


Since $\|F(s, \cdot)-f\| \leqq 2\|f\|$ and (4.16)holds, it follows from (4.19), (4.20) and condition $\mathrm{E}$ that $C(t, s)$ is strongly continuously differentiable in $t$ for some $s$. In fact, it follows that $C(t, s)$ is even uniformly differentiable and that $C_{t}(t, s)$ is even uniformly continuous.

5. Conditions implying $C_{3}$ for one exit or one regular boundary with reduced operator $\Omega(t)=D_{\mu(t)} D_{\sigma(t)}$.

LEMMA 5.1. (a) If $\Omega(t)=D_{\mu(t)} D_{\sigma(t)}$ and $r_{i}$ is an exit boundary and $r_{j}(i \neq j$; $i, j=1,2)$ is exit or natural, then conditions $\mathrm{A}, \mathrm{C}, \mathrm{F}_{1}, \mathrm{~F}_{2}, \mathrm{~F}_{3}, \mathrm{G}_{i}$ and $\mathrm{H}$ imply condition $\mathrm{C}_{3}: C(t, s)=[I-\Omega(t)][I-\Omega(s)]^{-1}$ is strongly continuously differentiable in $t$ for some s.

(b) If $\Omega(t)=D_{\mu(t)} D_{\sigma(t)}$ and $r_{i}$ is an exit boundary and $r_{j}(i \neq j ; i, j=1,2)$ is regular or entrance, then conditions $\mathrm{A}, \mathrm{C}, \mathrm{F}_{i}, \mathrm{~F}_{3}, \mathrm{G}_{i}$ and $\mathrm{H}$ imply condition $\mathrm{C}_{3}$.

In both case (a) and case (b) the domain of $\Omega(t)$ is independent of $t$ (Lemma (3.2)) so as in $\S 4, C(t, s)$ is in an endomorphism on $X$ for each $t, s$. Fix $s$ at that value for which $\mathrm{E}$ and $\mathrm{H}$ hold. As before for $f \in X$, we have equations (4.2) and (4.3). The proof of Lemma (5.1) proceeds in much the same manner as the proof of Lemma (4.1). Again we nominate a candidate $C_{t}(t, s)$ for the strong continuous derivative of $C(t, s)$. Again, we show that $C_{t}(t, s)$ is an element of $E(X)$, is the strong derivative of $C(t, s)$, and is such that $C_{t}(t, s)$ is strongly continuous.

In this case, however, we work with a different expression for $C_{t}(t, s) f$ obtained by replacing $D_{\sigma(s)} F(s, \cdot)$ in (4.3) by an equivalent taken from equation (4.17). Explicitly, from (4.17) we have,

$$
D_{\sigma(s)} F(s, x)=D_{\sigma(s)} F(s, 0)+\int_{0}^{x}[F(s, y)-f(y)] d \mu_{s} .
$$

So (4.3) yields the following expression for $C_{t}(t, s) f(x)$ :

$$
\begin{aligned}
C_{t}(t, s) f(x)= & -\psi_{t}(x, t, s)[F(s, x)-f(x)] \\
& -\phi_{t}(x, t, s) D_{\sigma(s)} F(s, 0) \\
& -\phi_{t}(x, t, s) \int_{0}^{x}[F(s, y)-f(y)] d \mu_{s} .
\end{aligned}
$$

Again the proof of the boundedness of $C_{t}(t, s)$ for the fixed $s$ poses the biggest problem. In demonstrating this boundedness we integrate equation (5.1) over $\left(x, r_{2}\right]$ where $x \in\left(0, r_{2}\right)$ is equal to the $x_{2}$ of condition $G_{2}$ (analogous considerations may be made for $r_{1}$ ) and obtain

$$
\begin{aligned}
\lim _{y \rightarrow r_{2}} F(s, y)-F(s, x)= & \int_{x}^{r_{2}} D_{\sigma(s)} F(s, 0) d \sigma_{s} \\
& +\int_{x}^{r_{2}} \int_{0}^{x}[F(s, y)-f(y)] d \mu_{s} d \sigma_{s} .
\end{aligned}
$$


Since $r_{2}$ is exit, the integrals on the right of (5.3) exist and $\lim _{y \rightarrow r_{2}} F(s, y)=0$. So (5.3) yields

$$
D_{\sigma(s)} F(s, 0)=\frac{-F(s, x)-\int_{x}^{r} \int_{0}^{x}[F(s, y)-f(y)] d \mu_{s} d \sigma_{s}}{\int_{x}^{r_{2}} d \sigma_{s}} .
$$

This expression for $D_{\sigma(s)} F(s, 0)$ can be used in (5.2), and the hypotheses of the lemma are readily seen to force the boundedness of $C_{t}(t, s)$ for the fixed $s$. If $r_{1}$ is the exit boundary instead of $r_{2}$, we integrate equation (5.1) over $\left[r_{1}, x\right)$ where $x \in\left(r_{1}, 0\right)$ is the $x_{1}$ of condition $\mathrm{G}_{1}$.

The fact that $C_{t}(t, s)$ is the strong derivative of $C(t, s)$ and is strongly continuous follows directly from (5.2) since $\psi$ and $\phi$ are strongly continuously differentiable.

As in Lemma 4.1, the method of proof used shows that $C_{t}(t, s)$ is the uniform derivative of $C(t, s)$ and that $C_{t}(t, s)$ is uniformly continuous.

CoRollary. (a) If $\Omega(t)=D_{\mu(t)} D_{\sigma(t)}$ and $r_{i}$ is regular, and if $r_{j}(i \neq j ; i,=1,2)$ is exit or natural, then conditions $\mathrm{A}, \mathrm{C}, \mathrm{F}_{j}, \mathrm{~F}_{3}, \mathrm{G}_{i}$ and $\mathrm{H}$ imply $\mathrm{C}_{3}$.

(b) If $\Omega(t)=D_{\mu(t)} D_{\sigma(t)}$ and $r_{i}$ is regular, and if $r_{j}(i \neq j ; i, j=1,2)$ is regular or entrance, then conditions $\mathrm{A}, \mathrm{C}, \mathrm{F}_{3}, \mathrm{G}_{i}$, and $\mathrm{H}$ imply $\mathrm{C}_{3}$.

Here equation (5.4) is replaced by

$$
D_{\sigma(s)} F(s, 0)=\frac{F\left(s, r_{2}\right)-F(s, x)-\int_{x}^{r} \int_{0}^{x}[F(s, y)-f(y)] d \mu_{s} d \sigma_{s}}{\int_{x}^{r_{2}} d \sigma_{s}}
$$

and the proof is analogous to that of Lemma 5.1.

6. The general operator. Let us now direct our attention to the general operator

$$
\Omega(t)=D_{\mu(t)} D_{\sigma(t)}+c(x, t)
$$

where $c(x, t)$ satisfies condition $\mathrm{D}$ : for each $t, c(\cdot, t) \leqq 0$ and is continuous on the closed interval $\left[r_{1}, r_{2}\right]$, and $c(\cdot, t)$ is strongly continuously differentiable in $t$ with strong derivative $c_{t}(\cdot, t) \in X$ for each $t$. By slightly modifying the proofs of Lemmas 3.1, 3.2, 4.1 and 5.1, one can readily prove that these lemmas are also true for the general operator $(6.1)$. Indeed if $c(\cdot, t) \leqq 0$, the key equations used in the proofs of these lemmas are modified by the addition of one to three terms involving $c(\cdot, t)$, and these additional terms fall in line with the proofs presented by virtue of the fact that $c(x, t)$ satisfies condition $D$. For example, if $c(\cdot, t) \neq 0$, equation (3.3) is replaced by

$$
\Omega(s) u=\psi(\cdot, s, t) D_{\mu(t)} D_{\sigma(t)} u+\phi(\cdot, s, t) D_{\sigma(t)} u+c(\cdot, s) u,
$$

and the key expressions for $C(t, s) f$ and $C_{t}(t, s) f$ become

$$
\begin{aligned}
C(t, s) f= & F(s, \cdot)-\psi(\cdot, t, s)[F(s, \cdot)-f]-\phi(\cdot, t, s) D_{\sigma(s)} F(s, \cdot) \\
& +\psi(\cdot, t, s) c(\cdot, s) F(s, \cdot)-c(\cdot, t) F(s, \cdot)
\end{aligned}
$$


and

$$
\begin{aligned}
C_{t}(t, s) f= & -\psi_{t}(\cdot, t, s)[F(s, \cdot)-f]-\phi_{t}(\cdot, t, s) D_{\sigma(s)} F(s, \cdot) \\
& +\psi_{t}(\cdot, t, s) c(\cdot, s) F(s, \cdot)-c_{t}(\cdot, t) F(s, \cdot) .
\end{aligned}
$$

In summary we see that the following theorem is true.

THEOREM (6.1). If $r_{1}$ and $r_{2}$ are each regular, entrance, or exit boundaries, or if one boundary is exit and one natural, or one is regular and one natural, then in each case any appropriate set of conditions given in Lemmas (4.1) and (5.1) which implies that $C(t, s)=[I-\Omega(t)][I-\Omega(s)]^{-1}$ is strongly continously differentiable in $t$ for some $s$ also implies that there exists a solution of the equation

$$
u_{t}(x, t)=D_{\mu(t)} D_{\sigma(t)} u(x, t)+c(x, t) u(x, t)
$$

where for each $t \in[a, b], u(\cdot, t)$ is an element of the Banach space $X$ of Definition 2.1 and where $c(x, t)$ satisfies condition $\mathrm{D}$. The solution is uniquely determined by the initial value $u(x, a)$ in $X$.

7. The special case of $A(t)=a(x, t) d^{2} / d x^{2}+b(x, t) d / d x$. Let us now consider the special case of the operator $A(t)=a(x, t) d^{2} / d x^{2}+b(x, t) d / d x$ where for each $t, a(x, t)>0$ and $b^{*}(x, t)=b(x, t) / a(x, t)$ is continuous on the open interval $\left(r_{1}, r_{2}\right)$ with $0 \in\left(r_{1}, r_{2}\right)$. In particular let us see what the boundary classifications and boundary conditions look like in this special case, and let us discover what restrictions the conditions of Theorem (6.1) impose on the coefficients of the equation $u_{t}(x, t)=A(t) u(x, t)$.

As in $\$ 2$ let

and

$$
\begin{aligned}
& B(x, t)=\int_{0}^{x} b^{*}(s, t) d s, \\
& \sigma(x, t)=\int_{0}^{x} e^{-B(s, t)} d s
\end{aligned}
$$

$$
\mu(x, t)=\int_{0}^{x} \frac{1}{a(s, t)} e^{B(s, t)} d s .
$$

Both $\sigma(x, t)$ and $\mu(x, t)$ are obviously monotone strictly increasing and $\sigma(x, t)$ is continuous on $\left(r_{1}, r_{2}\right)$ for each $t$ since $b^{*}(\cdot, t) \in C\left(r_{1}, r_{2}\right)$ for each $t$. The $J_{i}(t)$ and the $K_{i}(t)$ used in the boundary classifications become

and

$$
J_{i}(t)=(-1)^{i} \int_{0}^{r i} e^{-B(x, t)} \int_{0}^{x} \frac{1}{a(s, t)} e^{B(s, t)} d s d x
$$

$$
K_{i}(t)=(-1)^{i} \int_{0}^{r t} \frac{1}{a(x, t)} e^{B(x, t)} \int_{0}^{x} e^{-B(s, t)} d s d x
$$

In this case for each $t$ 
and

$$
K_{i}(t)<+\infty \rightarrow(-1)^{i} \int_{0}^{r t} \frac{1}{a(x, t)} e^{B(x, t)} d x<+\infty
$$

$$
J_{i}(t)<+\infty \rightarrow(-1)^{i} \int_{0}^{r t} e^{-B(x, t)} d x<+\infty .
$$

The classical boundary conditions become

$$
\begin{aligned}
\lim _{x \rightarrow r i}\left\{\left(1-p_{i}\right) u(x, t)+(-1)^{i} p_{i} u_{x}(x, t) e^{B(x, t)}\right\} & =0 \text { for } r_{i} \text { regular, } \\
\lim _{x \rightarrow r t} u(x, t) & =0 \text { for } r_{i} \text { exit, } \\
\lim _{x \rightarrow r i} u_{x}(x, t) e^{B(x, t)} & =0 \text { for } r_{i} \text { entrance, } \\
\lim _{x \rightarrow r i} u(x, t) & =0 \text { for } r_{i} \text { natural }
\end{aligned}
$$

where $i=1$ or 2 and $p_{i} \in[0,1]$.

From equations (7.2) and (7.3) we see that conditions $B, A$, and $F_{3}$ stated in $\$ 2$ are satisfied and in fact

Consequently

$$
\begin{aligned}
& \frac{d \mu_{s}}{d \sigma_{s}}=\frac{1}{a(x, s)} e^{2 B(x, s)}, \\
& \frac{d \mu_{t}}{d \mu_{s}}=\frac{a(x, s)}{a(x, t)} e^{B(x, t)} e^{-B(x, s)},
\end{aligned}
$$

$$
\frac{d \sigma_{t}}{d \sigma_{s}}=e^{B(x, s)} e^{-B(x, t)}
$$

$$
\begin{aligned}
\phi(\cdot, s, t) & =a(\cdot, s) e^{-B(\cdot, t)}\left[b^{*}(\cdot, s)-b^{*}(\cdot, t)\right], \\
\psi(\cdot, s, t) & =\frac{a(\cdot, s)}{a(\cdot, t)} \\
\phi(\cdot, s, t) \int_{0}^{x} d \mu_{t} & =\phi(\cdot, s, t) \int_{0}^{x} \frac{1}{a(v, t)} e^{B(v, t)} d v
\end{aligned}
$$

$$
\int_{x_{0}^{2}}^{r} d \sigma_{s}=\int_{x_{0}^{2}}^{r} e^{-B(x, s)} d x \text {. }
$$

Define the condition

$\mathrm{A}_{0}$. For each $t, a(\cdot, t)$ is positive and $b^{*}(\cdot, t)$ is continuous on $\left(r_{1}, r_{2}\right)$.

Then referring to the conditions $C_{1}, C_{2}, C_{3}$ listed in $\$ 1$ and the conditions on $\mu_{t}$ and $\sigma_{t}$ listed in $\S 2$, the main results established for $\Omega(t)=D_{\mu(t)} D_{\sigma(t)}$ may be specialized for $A(t)=a(x, t) d^{2} / d x^{2}+b(x, t) d / d x$ as follows.

LEMMA 7.1. In all boundary classifications condition $\mathrm{A}_{0}$ implies condition $\mathrm{C}_{1}: A(t)$ is defined for $t \in[a, b]$ and has property $S$ (Definition (1.1)) for each $t$. 
LEMMA 7.2. If both boundaries are regular, both are entrance, or one boundary is regular and one is entrance, $\mathrm{A}_{0}$ and $\mathrm{C}$ imply $\mathrm{C}_{2}$ : the domain of $A(t)$ is independent of $t$; and $\mathrm{A}_{0}, \mathrm{C}$ and $\mathrm{E}$ imply condition $\mathrm{C}_{3}: C(t, s)=[I-A(t)]$ $\cdot[I-A(s)]^{-1}$ is strongly continuously $d$ ifferentiable in $t$ for some $s$.

LEMMA 7.3. (a) If both boundaries are exit, both are natural, or one boundary is natural and one is exit, $\mathrm{A}_{0}, \mathrm{C}, \mathrm{F}_{1}$ and $\mathrm{F}_{2}$ imply $\mathrm{C}_{2}$. If $r_{i}$ is exit and $r_{j}$ $(i \neq j ; i, j=1,2)$ is exit or natural, $\mathrm{A}_{0}, \mathrm{C}, \mathrm{F}_{1}, \mathrm{~F}_{2}, \mathrm{G}_{i}$ and $\mathrm{H}$ imply $\mathrm{C}_{3}$.

(b) If $r_{i}$ is natural or exit and if $r_{j}(i \neq j ; i, j=1,2)$ is regular or entrance, $\mathrm{A}_{0}, \mathrm{C}$ and $\mathrm{F}_{i}$ imply $\mathrm{C}_{2}$. If $r_{i}$ is exit and $r_{j}$ is regular or entrance, $\mathrm{A}_{0}, \mathrm{C}, \mathrm{F}_{i}, \mathrm{G}$ and $\mathrm{H}$ imply $\mathrm{C}_{3}$.

LEMMA 7.4. (a) If $r_{i}$ is regular and if $r_{j}(i \neq j)$ is exit or natural, $\mathrm{A}_{0}, \mathrm{C}$, $\mathrm{F}_{j}, \mathrm{G}_{i}$ and $\mathrm{H}$ imply $\mathrm{C}_{3}$.

(b) If $r_{i}$ is regular and if $r_{j}$ is regular or entrance, $\mathrm{A}_{0}, \mathrm{C}, \mathrm{E}$ and $\mathrm{G}_{i}$ imply $\mathrm{C}_{3}$. In each case each appropriate set of conditions implying $\mathrm{C}_{3}$ implies the existence of a solution of the equation

$$
u_{t}(x, t)=a(x, t) u_{x x}(x, t)+b(x, t) u_{x}(x, t)
$$

uniquely determined by the initial value $u(x, a)$. Of course if $c(x, t)$ satisfies condition $\mathrm{D}$, then each set of conditions implying $\mathrm{C}_{3}$ is also a set of conditions implying the existence of a solution of the equation

$$
u_{t}(x, t)=a(x, t) u_{x x}(x, t)+b(x, t) u_{x}(x, t)+c(x, t) u(x, t)
$$

uniquely determined by $u(x, a)$.

8. Examples. In each specific example it is necessary to determine whether or not the strong derivatives $\phi_{t}(\cdot, t, s)$ and $\psi_{t}(\cdot, t, s)$ exist. This computation is frequently made much easier by using a simple criterion. This criterion asserts that if $\partial^{2} g / \partial t^{2}$ is continuous on $\left[r_{1}, r_{2}\right]$ and bounded uniformly in $x$ and $t$, then the derivative $\partial g / \partial t$ exists as a strong derivative.

EXAMPLE 1. As an example of a particular equation $u_{t}=A(t) u$ with coefficients which satisfy the conditions of Theorem (6.1) even though $b(x, t)$ is discontinuous at both boundaries, consider the equation

$$
u_{t}(x, t)=u_{x x}(x, t)+\left(\frac{-x}{1-x^{2}}+3 t\left(1-x^{2}\right)\right) u_{x}(x, t)
$$

for $x \in[-1,1]$. Here

$$
e^{B(x, t)}=\left(1-x^{2}\right)^{1 / 2} e^{t\left(3 x-x^{3}\right)} .
$$

We see that both $e^{B(x, t)}$ and $e^{-B(x, t)}$ are in $L(0,1)$ and in $L(-1,0)$ so that 1 and -1 are both regular boundaries. Choose the boundary conditions so that $X$ is $C[-1,1]$. 
$b(x, t)$ is clearly continuous on the open interval $(-1,1)$ for each $t$ so condition A (82) is satisfied. We have

$$
\phi(x, s, t)=3\left(1-x^{2}\right)^{1 / 2} e^{-t\left(3 x-x^{3}\right)}(s-t) \text { and } \psi(x, t, s) \equiv \psi(x, s, t) \equiv 1 .
$$

$\phi(\cdot, s, t)$ and $\psi(\cdot, s, t)$ are both continuous on the closed interval $[-1,1]$ so condition $\mathrm{C}(\S 2)$ is satisfied. Both $\phi_{t}(\cdot, t, s)$ and $\psi_{t}(\cdot, t, s)$ are in $C[-1,1]$ for the fixed $s$ and condition $\mathrm{E}(\$ 2)$ is satisfied.

EXAMPLE 2. An example of the case in which $b(x, t)$ is discontinuous at an entrance boundary is given by the equation

$$
u_{t}(x, t)=u_{x x}(x, t)+\left(\frac{1}{x-1}+2 h(t)(x-1)\right) u_{x}(x, t)
$$

where $x \in[-1,1], h(t) \geqq 0$ for each $t \in[a, b]$, and $d^{2} h / d t^{2}$ is continuous. Here

$$
e^{B(x, t)}=(x-1) e^{h(t)\left(x^{2}-2 x\right)} \text {. }
$$

Clearly $e^{B(x, t)}$ is an element of both $L(-1,0)$ and $L(0,1)$. Also $e^{-B(x, t)} \in L(-1,0)$. However, $e^{-B(x, t)}$ is not an element of $L(0,1)$. Thus, -1 is a regular boundary and +1 is either entrance or natural. In fact, it is readily seen that $e^{B(x, t)} \int_{0}^{x} e^{-B(y, t)} d y$ is in $L(0,1)$ so +1 is an entrance boundary.

Condition $A_{0}(\$ 7)$ is certainly true, and

$$
\begin{aligned}
& \phi(x, s, t)=2 e^{-h(t)\left(x^{2}-2 x\right)}[h(s)-h(t)], \\
& \psi(x, s, t) \equiv \psi(x, t, s) \equiv 1 .
\end{aligned}
$$

So, if we choose the boundary conditions so that $X$ is $C[-1,1], \phi(\cdot, s, t)$ and $\psi(\cdot, s, t)$ are both in $X$ for each $s, t$. Also for fixed $s$, by the strong differentiability criterion

and

$$
\phi_{t}(x, t, s)=2 e^{-h(s)\left(x^{2}-2 x\right)} h_{t}(t)
$$

$$
\psi_{t}(\cdot, t, s)=0
$$

so $\phi_{t}(\cdot, t, s)$ and $\psi_{t}(\cdot, t, s)$ are both in $X$ for each $t$ and the fixed $s$. It follows that there exists a solution of (8.2) uniquely determined by the initial value.

EXAmple 3. The case in which $b(x, t)$ is discontinuous at an exit boundary may be illustrated by the equation

$$
u_{t}(x, t)=u_{x x}(x, t)+\left(\frac{1}{1-x}+2 h(t)(1-x)\right) u_{x}(x, t)
$$

where $x \in[-1,1], t \in[a, b], a \geqq 0$, and $h(t) \geqq 0$ for each $t$ with $d^{2} h / d t^{2}$ continuous. Here both $e^{B(x, t)}$ and $e^{-\bar{B}(x, t)}$ are elements of $L(-1,0)$ so -1 is regular. $e^{-B(x, t)}$ is also an element of $L(0,1)$, but $e^{B(x, t)} \notin L(0,1)$. Thus +1 is either exit or natural. Since it may be shown that $\int_{0}^{1} e^{-B(x, t)} \int_{0}^{x} e^{B(y, t)} d y d x<\infty,+1$ is actually an exit boundary. 
The conditions which must be true in order for Theorem (6.1) to hold for equation (8.3) are $A_{0}(\$ 7)$, and $C, E, F_{2}, G_{1}$ and $H(\$ 2)$. It may be readily shown that these conditions are all true in this example.

EXAMPLE 4. Now let us examine an example of an operator $A(t)$ whose coefficient $b(x, t)$ has a jump discontinuity at some point within $\left[r_{1}, r_{2}\right]$ but which is such that the existence and uniqueness of the solution of $u_{t}=A(t) u$ with initial value $u(x, a)$ can be ascertained without piecing together two solutions. Let $x \in[-1,1]$ and $t \in[a, b], a \geqq 0$. Let

$$
\begin{aligned}
u_{t}(x, t) & =u_{x x}(x, t)+2 x t u_{x}(x, t) & & \text { for } x<0 \\
& =u_{x x}(x, t)+(2 x t+3) u_{x}(x, t) & & \text { for } x \geqq 0 .
\end{aligned}
$$

Then

$$
\begin{aligned}
B(x, t) & =x^{2} t & & \text { for } x<0 \\
& =x^{2} t+3 x & & \text { for } x \geqq 0 .
\end{aligned}
$$

We see that $e^{B(x, t)}$ and $e^{-B(x, t)}$ are both in $L(0,1)$ and $L(-1,0)$ so -1 and 1 are both regular boundaries. Let

$$
\begin{aligned}
\sigma_{t}(x) & =\int_{0}^{x} e^{-y^{2} t} d y & & \text { for } x<0, \\
& =\int_{0}^{x} e^{-y^{2} t-3 y} d y & & \text { for } x \geqq 0, \\
\mu(x, t) & =\int_{0}^{x} e^{y^{2} t} d y & & \text { for } x<0, \\
& =\int_{0}^{x} e^{y^{2} t+3 y} d y & & \text { for } x \geqq 0 .
\end{aligned}
$$

Then (8.4) is reduced to the canonical form

$$
u_{t}=D_{\mu(t)} D_{\sigma(t)} u
$$

which holds for all $x \in[-1,1]$.

Note that conditions A and B (§2) are both true. Further

$$
\begin{array}{rlrl}
\phi(x, s, t) & =2 x e^{-x^{2} t}(s-t) & \text { for } x<0 \\
& =2 x e^{-x^{2} t-3 x}(s-t) & \text { for } x \geqq 0 \\
\psi(x, s, t) \equiv 1 & \text { for } x \in[-1,1] \text { and } s, t \in[a, b] .
\end{array}
$$

Note that $\lim _{x \rightarrow 0^{+}} \phi(x, s, t)=\lim _{x \rightarrow 0^{-}} \phi(x, s, t)=\phi(0, s, t)=0$ so $\phi(\cdot, s, t)$ and $\psi(\cdot, s, t)$ are both continuous for all $x \in[-1,1]$ for each $s, t$. Thus, if we choose the regular boundary conditions so that $X$ is $C[-1,1]$, condition $C(\S 2)$ is true.

For fixed $s$ we have 
and

$$
\begin{aligned}
\phi_{t}(x, t, s) & =2 x e^{-x^{2} s} & & \text { for } x<0 \\
& =2 x e^{-x^{2} s-3 x} & & \text { for } x \geqq 0
\end{aligned}
$$

$$
\psi_{t}(x, t, s) \equiv 0 .
$$

We see that for the fixed $s, \phi_{t}(\cdot, t, s)$ and $\psi_{t}(\cdot, t, s)$ are both continuous for all $x \in[-1,1]$ for each $t$ so condition $\mathrm{E}(\$ 2)$ is satisfied. It follows that there exists a solution to (8.4) uniquely determined by $u(x, a)$.

\section{REFERENCES}

1. J. Elliott, Eigenfunction expansions associated with certain singular differential operators, Trans. Amer. Math. Soc. 78 (1955), 406-425.

2. W. Feller, On differential operators and boundary conditions, Comm. Pure Appl. Math. 8 (1955), 203-216.

3. - On second order differential operators, Ann. of Math. 61 (1955), 90-105.

4. - The parabolic differential equations and the associated semi-groups of transformations, Ann. of Math. 55 (1952), 468-519.

5. - Zur Theorie der stochastichen Prozesse, Math. Ann. 112 (1936), 113-160.

6. E. Hille, Functional analysis and semi-groups, Amer. Math. Soc. Colloq. Publ. Vol. 31, Amer. Math. Soc., Providence, R.I., 1948.

7. - The abstract Cauchy problem and Cauchy's problem for parabolic differential equations, J. Analyse Math. 3 (1954), 81-196.

8. E. Hille and R. S. Phillips, Functional analysis and semi-groups, Amer. Math. Soc. Colloq. Publ. Vol. 31, Amer. Math. Soc., Providence, R.I., 1957.

9. T. Kato, Integration of the equation of evolution in a Banach space, J. Math. Soc. Japan 5 (1953), 208-234.

10. - On linear differential equations in Banach spaces, Comm. Pure Appl. Math. 9 (1956), 479-486.

11. H. P. McKean, Jr., Elementary solutions for certain parabolic partial differential equations, Trans. Amer. Math. Soc. 82 (1945), 519-547.

12. D. G. S. Stockton, Singular parabolic partial differential equations with time dependent coefficients, University Microfilms Inc., L. C. Card No. Mic. 58-7667.

UNIVERSTTY OF MASSACHUSETTS, AMHERST, MASSACHUSETTS 